\title{
Real-time magnetic resonance imaging-guided frameless stereotactic brain biopsy: technical note
}

\author{
Ahmed Mohyeldin, MD, PhD, Russell R. Lonser, MD, and J. Bradley Elder, MD \\ Department of Neurological Surgery, The Ohio State University Wexner Medical Center, The Ohio State University \\ Comprehensive Cancer Center, Arthur G. James Cancer Hospital and Richard J. Solove Research Institute, Columbus, Ohio
}

\begin{abstract}
OBJECTIVE The object of this study was to assess the feasibility, accuracy, and safety of real-time MRI-compatible frameless stereotactic brain biopsy.

METHODS Clinical, imaging, and histological data in consecutive patients who underwent stereotactic brain biopsy using a frameless real-time MRI system were analyzed.
\end{abstract}

RESULTS Five consecutive patients (4 males, 1 female) were included in this study. The mean age at biopsy was 45.8 years (range 29-60 years). Real-time MRI permitted concurrent display of the biopsy cannula trajectory and tip during placement at the target. The mean target depth of biopsied lesions was $71.3 \mathrm{~mm}$ (range 60.4-80.4 mm). Targeting accuracy analysis revealed a mean radial error of $1.3 \pm 1.1 \mathrm{~mm}$ (mean \pm standard deviation), mean depth error of $0.7 \pm 0.3$ $\mathrm{mm}$, and a mean absolute tip error of $1.5 \pm 1.1 \mathrm{~mm}$. There was no correlation between target depth and absolute tip error (Pearson product-moment correlation coefficient, $r=0.22$ ). All biopsy cannulae were placed at the target with a single penetration and resulted in a diagnostic specimen in all cases. Histopathological evaluation of biopsy samples revealed dysembryoplastic neuroepithelial tumor (1 case), breast carcinoma (1 case), and glioblastoma multiforme (3 cases).

CONCLUSIONS The ability to place a biopsy cannula under real-time imaging guidance permits on-the-fly alterations in the cannula trajectory and/or tip placement. Real-time imaging during MRI-guided brain biopsy provides precise safe targeting of brain lesions.

http://thejns.org/doi/abs/10.3171/2015.5.JNS1589

KEY WORDS biopsy; technique; magnetic resonance imaging; tumor; surgical technique

$\mathrm{D}$ ESPITE their ubiquitous use, both frame-based and frameless stereotactic biopsies rely on preoperative images with intraoperative anatomical or fiducial registration to reach deep brain targets based on precalculated measurements in 3D space. ${ }^{1,3,6,8,14,15,23}$ The primary limitations of these approaches are the inability to account for the shift of intracranial structures (reduces accuracy of the biopsy at the target), the inability to adapt to vital structures along the needle trajectory in real-time, and the lack of intraoperative imaging confirmation of the accuracy of the biopsy target., ${ }^{5,13,22}$ Moreover, miscalculations or technical misalignment of hardware during biopsy can result in intraoperative morbidity. ${ }^{6,10}$ Recent data indicate that the diagnostic accuracy of stereotaxy using conventional preoperative neuronavigation may be lower than previously reported (accuracy range 51\%-91\%). $.3,8$

To overcome these limitations, technology has been developed that now permits the integration of intraoperative
MRI (iMRI) with frameless stereotaxy. The resolution and real-time feedback of this iMRI technology has been demonstrated in phantom experiments, ${ }^{12,18}$ nonhuman primate studies ${ }^{16,17}$ drug infusion cannula placement, ${ }^{2}$ and placement of deep brain stimulator leads. . $11,20,21^{\text {Application of }}$ this technology to stereotactic brain biopsy could eliminate misdiagnosis secondary to faulty targeting, provide realtime adaptability for needle trajectory, confirm accurate biopsy cannula position, and provide immediate imaging of complications. To determine the feasibility, accuracy, and safety of real-time MRI-guided brain biopsy, we analyzed the results in consecutive brain biopsy cases using this paradigm.

\section{Methods}

\section{Patients}

Consecutive patients who underwent stereotactic brain 
biopsy using frameless real-time MRI were included in this study. All patients consented to real-time stereotactic iMRI biopsy using the technique described below.

\section{Biopsy Platform}

The ClearPoint System (MRI Interventions Inc.) is composed of an integrated head fixation frame, a disposable SmartFrame scalp-mounted guide, a dedicated computer workstation for surgical planning, and an MR-compatible in-room computer monitor (Fig. 1). An MR-compatible cannula, including a ceramic guide stylet (MRI Interventions Inc.) and a biopsy needle (Ad-Tech) were used for targeting and tissue collection.

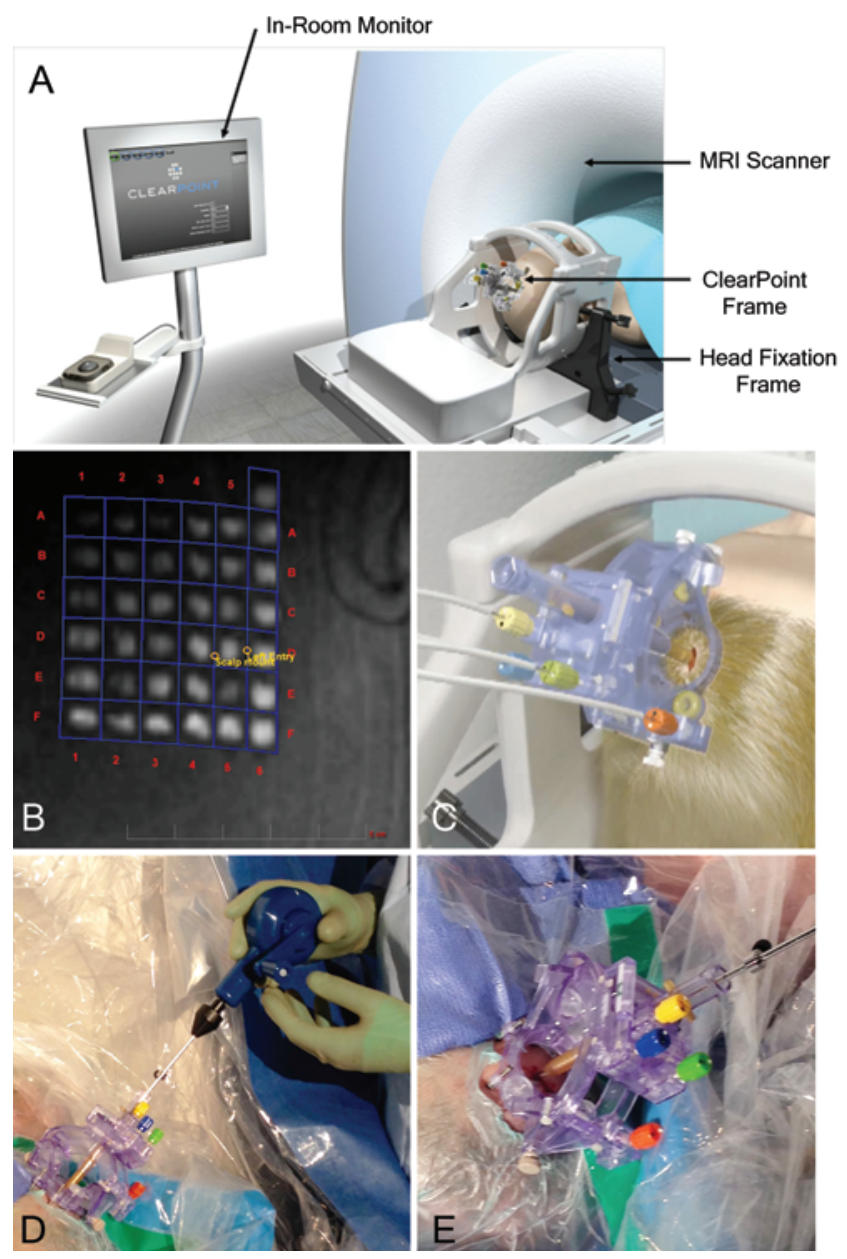

FIG. 1. The real-time MRI system includes integrated workflow for the performance of frameless stereotactic biopsy. A: Artist's depiction of surgical setup. The in-room monitor is positioned in the operative suite in relation to the patient and MR unit. Also depicted is the needle-guiding frame affixed to the patient's head. B: An MR image of the localizer grid affixed to the patient's scalp, which is used to define entry point and twist-drill hole placement. C: Artist's depiction of stereotactic device platform with detachable control knobs (colored) to guide trajectory adjustments after MR scan. D: Intraoperative placement of a twist-drill hole via a hand held drill performed percutaneously through the skin. E: Intraoperative image of the stereotactic device platform mounted on the patient's head before biopsy needle insertion. Images in A and B, copyright MRI Interventions. Published with permission. Figure is available in color online only.

\section{Imaging System and Operating Room}

An open "single-doughnut" system with a 1.5-T MR unit (Siemens AG) was used in all cases. The ClearPoint Workstation uses proprietary software (ClearPoint Imaging Software version 1.51) to communicate with the MRI console and permits the surgeon to make targeting decisions and adjustments in the operating room. The iMRI suite is a fully functional operating room with the same standards of airflow and sterility applied for regular operating suites at our institution. The suite is equipped with an MR-compatible anesthesia machine and monitoring devices. Magnetic resonance-compatible surgical instruments were used in all cases.

\section{Histopathological Analysis}

Core biopsy samples were sent for permanent tissue pathology evaluation. Routine and immunohistochemical studies were performed as indicated.

\section{Targeting Accuracy Data and Statistical Analysis}

The accuracy of biopsy needle insertion in relation to the intended target was quantified in patients by measuring the radial error and depth error, as well as by calculating the absolute tip error. The measurements needed to calculate these errors, target location, and tip location were obtained from images acquired during real-time MRI-guided biopsy. The intended target location was selected on initial images prior to placement of the biopsy needle. Location of the needle tip was manually selected by identifying the center-most distal tip of the needle on MRI sequences obtained prior to tissue harvest. The stereotactic software automatically reported vector distances between the intended target site and the actual position of the biopsy needle tip to give target depth, radial error, and depth error for each patient in 3D space (Fig. 2), and the primary author and senior author reviewed all data for analysis. The primary measure for error reporting was the absolute tip error, which was the calculated distance from the tip of the biopsy needle to the intended target. Error measurements were tested for linear correlation by calculating the Pearson product-moment correlation coefficient. A coefficient of 1 is considered a total positive correlation, 0 is no correlation, and -1 is a total negative correlation. ${ }^{19}$

\section{Technique}

Prior to surgery, the preoperative MRI sequences are loaded into the computer workstation, and the cannula entry point, trajectory, and target for biopsy are selected. After general anesthesia is induced, hair in the region of the entry point is shaved to accommodate the $4 \times 4-\mathrm{cm}$ localizer grid, which is placed once the patient is positioned in the MR facility. Patients are then taken into the MR facility and positioned in an MR-compatible head fixation frame with 4 points of fixation. Three patients in this study were supine and 2 were prone. Head rotation/ flexion/extension was adjusted in each case to optimize the trajectory of the biopsy needle relative to the surgical field and space limitations of the bore of the magnet. A T1weighted MR image is then obtained, and the anterior and posterior commissures are identified to orient the software 


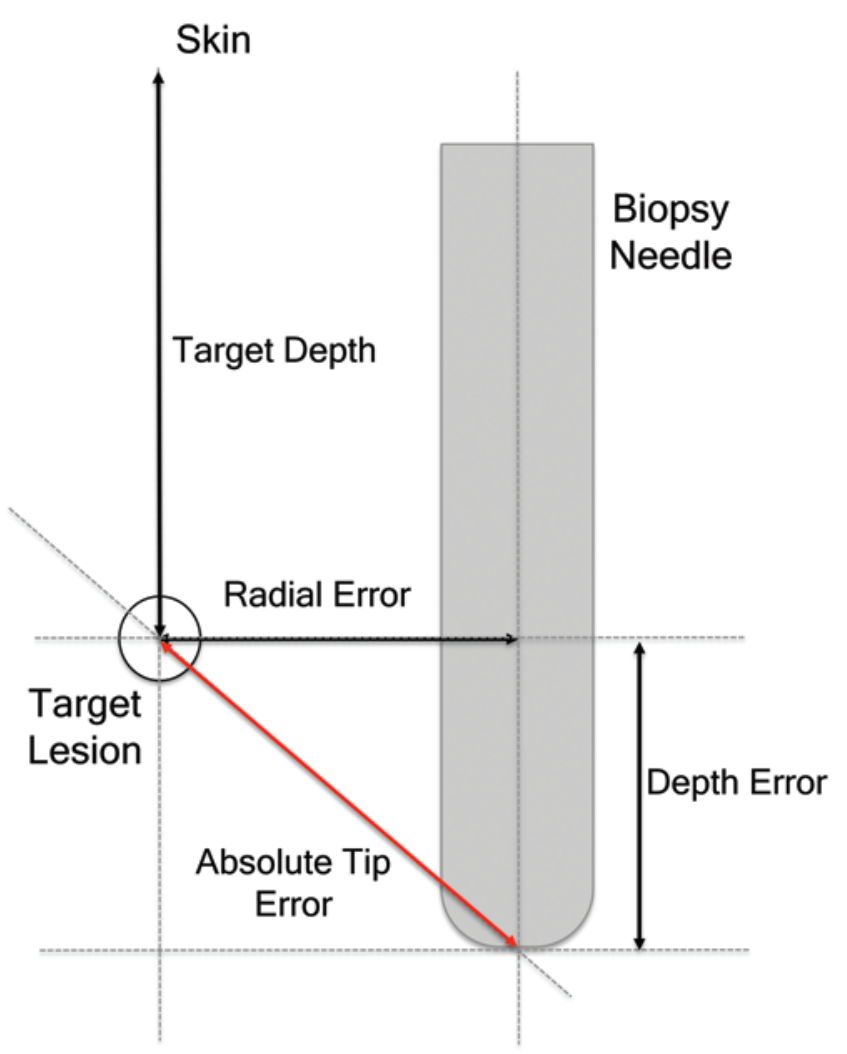

FIG. 2. Targeting accuracy was quantified by measuring the direct target depth, the radial error, and the depth error and by calculating the absolute tip error. Illustration depicting the conceptual relationship behind the various error values measured (black lines) and those that are calculated (red line) from the tip of the biopsy needle to the center of the preset target lesion. Figure is available in color online only.

system to the head position. After this initial image, the surgical site is prepared and draped using a specialized draping system compatible with multiple transitions into and out of the bore of the magnet.

A $4 \times 4-\mathrm{cm}$ localizer grid (Fig. 1) is then applied to the scalp, overlying the predetermined incision site and skull entry point. A T1-weighted MR image is obtained either without or with a half dose of gadolinium based on enhancing properties of the lesion (a half dose of gadolinium is used in enhancing lesions so that another dose of gadolinium can be used later if needed). Coordinates of the preselected entry point relative to the localizer grid are then determined using the integrated software at a computer workstation, and the final entry point, trajectory, and target points are confirmed. The localizer grids are then removed, and an MR-compatible biopsy frame is attached percutaneously (using $1.62-\mathrm{mm}$ screws) to the patient's skull, centered over the entry point as guided by the localizer grids (Fig. 1).

A series of T1-weighted MR images focused on the biopsy frame and target lesion are then used to fine-tune the trajectory of the guide cannula mounted on the frame. The software detects the position of the cannula relative to the base and target that were previously scanned and defined. This will provide a projection of where the cannula is currently aimed and give the user instructions to grossly align the cannula to the target. Between each scan, the biopsy system is fine-tuned as guided by the navigation system software. Specifically, a series of images are taken at oblique coronal and sagittal planes along the trajectory of the cannula (with isocentering of the scan parameters to optimize distortion-free representation). These data are overlaid, and a best-fit line is projected from the cannula to the target in MR space.

Once the trajectory is finalized, a percutaneous twistdrill hole is made in the skull, and a ceramic guide cannula (1.5-mm diameter) is inserted along the preselected trajectory to a depth $5 \mathrm{~mm}$ from the surface of the target lesion. Real-time T1-weighted MRI during cannula placement is employed to ensure accuracy of the cannula trajectory and safety of the pathway to the target. The ceramic stylet (1.5-mm diameter) is removed once the trajectory is confirmed. The biopsy needle $(2.1 \mathrm{~mm})$ is then inserted along the same trajectory to the appropriate depth within the lesion, and after confirming the final location of the biopsy needle with MRI, multiple core biopsy samples are harvested. Tissue cores are harvested using an MRIcompatible, side-cutting biopsy needle (Ad-Tech) and sent for permanent section.

After completing the biopsy core sampling, the biopsy needle is maintained in place for 5 minutes to assist with hemostasis and is then removed. Final MRI specific to the imaging properties of the lesion is then performed to evaluate for complications and identify any tissue defect and/ or needle tract, which secondarily verifies accuracy of the biopsy site. The cannula and guidance frame are then detached from the skull, and the percutaneous stab incisions are closed with a cutaneous adhesive agent (Dermabond, Ethicon).

\section{Results \\ Patients}

Five patients ( 4 males, 1 female) were included in the study. Mean age at surgery was 45.8 years (range 29-60 years). Patient characteristics are included in Table 1.

\section{Intraoperative Imaging Findings}

Intraoperatively, lesions varied in size and radiographic appearance based on contrast enhancement on T1-weighted imaging or increased FLAIR/T2 signal intensity (Table 1). The ceramic stylet was clearly visible during placement as a hypointense signal on T1-weighted imaging. Real-time imaging of the ceramic stylet confirmed an accurate trajectory and depth in all cases, and no targeting adjustments were required prior to placement of the biopsy needle. The biopsy needle was noted to have a larger hypointense signal diameter on T1-weighted sequences. Postbiopsy scans revealed T1- and T2-weighted hypointense signal changes within the lesion consistent with a defect created by tissue harvest.

\section{System Accuracy}

Intraoperative MRI confirmed accurate biopsy needle placement at the target in all patients. Mean target depth was $71.3 \mathrm{~mm}$ (range $60.4-80.4 \mathrm{~mm}$ ), mean radial error was $1.3 \pm 1.1 \mathrm{~mm}$ (mean \pm standard deviation), and mean depth error was $0.7 \pm 0.3 \mathrm{~mm}$. The mean absolute tip error 
TABLE 1. Features of 5 biopsy cases

\begin{tabular}{|c|c|c|c|c|c|c|c|}
\hline $\begin{array}{c}\text { Case } \\
\text { No. }\end{array}$ & Age (yrs) & Sex & Lesion Location & Lesion Size $(\mathrm{cm})^{\star}$ & Pathological Diagnosis & Length of Op (min) & Imaging Sequence $†$ \\
\hline 1 & 38 & $\mathrm{~F}$ & Left thalamic & 2.18 & Breast carcinoma & 177 & T1 \\
\hline 2 & 29 & M & Left frontal & 1.59 & DNET & 139 & $\mathrm{~T} 2 / \mathrm{T} 1$ \\
\hline 3 & 60 & M & Right parietal & 8.01 & GBM & 84 & T1 \\
\hline 4 & 52 & M & Left temporal & 2.02 & GBM & 94 & T1 \\
\hline 5 & 50 & M & Left temporal & 3.67 & GBM & 76 & $\mathrm{~T} 1$ \\
\hline
\end{tabular}

was $1.5 \pm 1.1 \mathrm{~mm}$ with a 95\% CI 0.51-1.49 (Table 2). Mean operative time was $114 \pm 42.8$ minutes. Biopsied tissue provided a definitive diagnosis in all cases (Table 1). All biopsy needles were placed with a single brain penetration. No correlation was found between the target depth and the absolute tip error (calculated Pearson product-moment correlation coefficient, $r=0.22$ ).

\section{Complications}

There were no surgical complications in any of the patients. No evidence of a bleed was noted on intraoperative imaging.

\section{Illustrated Cases \\ Case 1}

A 38-year-old female with a history of breast cancer presented with new-onset seizures (Table 1). Magnetic resonance imaging revealed a left posterior thalamic enhancing mass (Fig. 3). Given the depth of the lesion and its proximity to the brainstem and adjacent white fiber tracts, real-time iMRI-guided biopsy was performed to obtain tissue diagnosis before radiation therapy. Intraoperative imaging was performed to determine the trajectory to the target and biopsy planning. Using real-time T1-weighted MRI, we placed the biopsy needle within the enhancing lesion. Magnetic resonance imaging confirmed the presence of the biopsy needle tip in the lesion, and a biopsy was performed. Histological diagnosis revealed metastatic breast carcinoma.

\section{Case 2}

A 29-year-old male (Table 1) presented with a generalized tonic-clonic seizure. An abnormal focus of hyperintensity on T2-weighted MRI was demonstrated within the left inferior frontal lobe that did not contrast enhance (Fig. 4). Given the small size of the lesion and its proximity to the anterior cerebral artery, the lesion was approached for biopsy via a real-time iMRI-guided approach. Intraoperative imaging was performed to determine a trajectory, which avoided the lateral ventricle and corpus callosum. Using real-time T1-weighted MRI, we placed the ceramic cannula $5 \mathrm{~mm}$ above the target to confirm the trajectory. Magnetic resonance imaging confirmed the presence of the biopsy needle tip at the target, and a biopsy was performed. Histological diagnosis revealed dysembryoplastic neuroepithelial tumor (DNET). Intraoperative T2-weight- ed MRI confirmed accurate targeting, as evidenced by a cavity at the center of the lesion.

\section{Case 3}

A 60-year-old male presented with a recurrent right occipital lobe glioblastoma multiforme (GBM). The patient was symptomatic with gait imbalance, a visual field cut, headaches, and emesis. Magnetic resonance imaging revealed possible tumor recurrence or pseudo-progression. It also revealed increased periventricular contrast enhancement along the anterior margin of the resection cavity that extended into the splenium of the corpus callosum. Magnetic resonance perfusion imaging demonstrated a small area of hyperperfusion within the lesion. An iMRI-guided biopsy was preferred over conventional stereotactic biopsy to target the small focus of hyperperfusion, which had a unique enhancement profile compared with surrounding tissues. Intraoperative MR images confirmed accurate placement of the biopsy cannula in the target area of the lesion (captured with T1-weighted images with contrast and correlated with the perfusion imaging). Multiple cores were harvested and sent for permanent section, which confirmed a diagnosis of recurrent GBM.

\section{Case 4}

A 52-year-old male with a known history of high-grade glioma presented with possible tumor recurrence. Magnetic resonance imaging revealed a small left temporal enhancing nodule adjacent to the previous resection cavity but close to the brainstem and surrounding critical basal cistern vasculature. An iMRI-guided biopsy was preferred over conventional stereotactic biopsy for accurate target-

TABLE 2. Targeting accuracy measurements

\begin{tabular}{ccccc}
\hline Parameter & $\begin{array}{c}\text { Target } \\
\text { Depth }(\mathrm{mm})\end{array}$ & $\begin{array}{c}\text { Radial Error } \\
(\mathrm{mm})\end{array}$ & $\begin{array}{c}\text { Depth Error } \\
(\mathrm{mm})\end{array}$ & $\begin{array}{c}\text { Absolute Tip } \\
\text { Error }(\mathrm{mm})\end{array}$ \\
\hline Case No. & & & & \\
\hline 1 & 74.8 & 3.3 & 1.1 & 3.5 \\
\hline 2 & 80.4 & 0.5 & 0.6 & 0.8 \\
\hline 3 & 60.4 & 0.8 & 0.4 & 0.9 \\
\hline 4 & 71.5 & 0.8 & 0.6 & 1.0 \\
\hline 5 & 69.4 & 1.0 & 0.9 & 1.3 \\
\hline Average & 71.3 & 1.3 & 0.7 & 1.5 \\
\hline SD & 7.4 & 1.1 & 0.3 & 1.1 \\
\hline
\end{tabular}




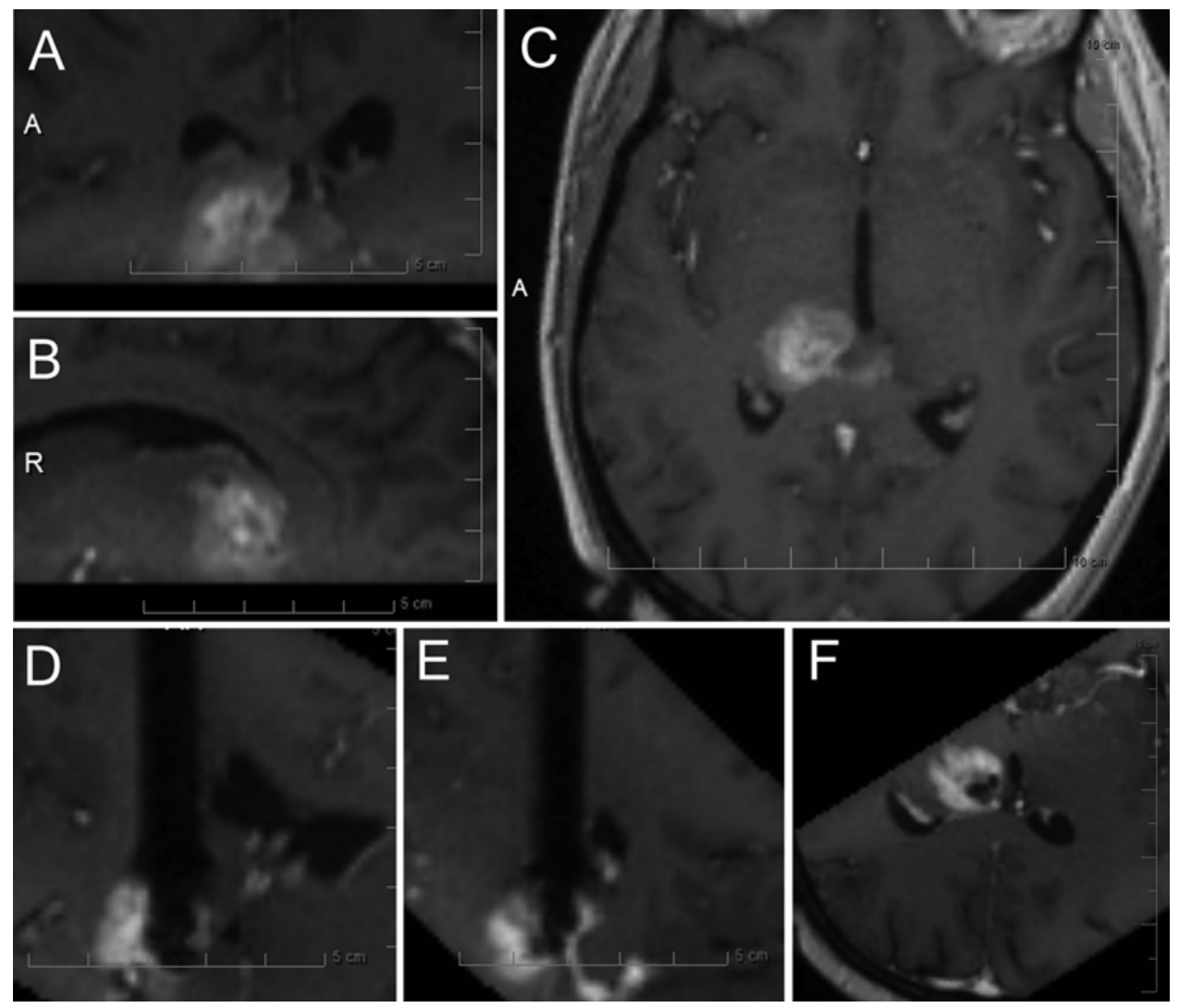

FIG. 3. Case 1. Intraoperative MR images obtained with a 1.5-T scanner. Preoperative coronal (A), sagittal (B), and axial (C) T1-weighted MR images obtained with contrast for trajectory planning. Intraoperative coronal (D) and sagittal (E) T1-weighted MR images with contrast demonstrate surrounding vascular structures and insertion of the biopsy needle through the guide cannula. Trajectory view (F) using T1-weighted MR image with cutting window, showing the biopsy needle at the target in the lesion before tissue sampling.

ing of the small focus of enhancement in this eloquent region. Intraoperative MR images to confirm accurate placement of the cannula in the target lesion were captured using T1-weighted sequences with contrast. Multiple cores were harvested and sent for pathology and confirmed a diagnosis of GBM.

\section{Case 5}

A 50-year-old male with a known history of high-grade glioma presented with aphasia and imaging evidence of possible tumor recurrence. An iMRI-guided biopsy was selected over conventional stereotactic biopsy for the most accurate targeting of the small focus of enhancement in the insular region. Intraoperative images to confirm accurate placement of the cannula in the target lesion were captured using T1-weighted sequences with contrast. Multiple cores were harvested and sent for pathology and confirmed a diagnosis of GBM.

\section{Discussion}

\section{Previous Work}

Recently, this frameless stereotactic guidance system has been successfully used for accurate real-time MRIguided placement of deep brain stimulator leads, ${ }^{9,20,21}$ laser diode applicators, ${ }^{24}$ and convection-enhanced-delivery infusion cannulae. ${ }^{16,17}$ Given the backdrop of applications, this frameless stereotactic guidance system offers an opportunity to perform real-time MRI-guided biopsy with advantages over currently available stereotactic biopsy techniques. Specifically, this platform can be used via a percutaneous approach, provides real-time confirmation of trajectory or target accuracy, and is compatible with high-field magnets that provide for excellent resolution. Because real-time imaging is performed during cannula and/or biopsy needle placement, intraoperative trajectory and/or target adjustments are possible.

Here, we assessed the feasibility, accuracy, and safety of this real-time MRI-guided brain biopsy platform in 5 consecutive brain biopsy cases.

\section{Current Study}

\section{Patients}

While this technology has potential advantages for use in all biopsies, the patients in the current report were selected to undergo iMRI biopsy for brain lesions or for targets that were small and/or located in anatomical regions near critical vascular and/or anatomical structures. Consequently, these cases illustrate the safety and accuracy of this technique, as well as underscore the potential advantage for sampling lesions in eloquent anatomical locations or areas otherwise potentially difficult to reliably target (Table 1). The real-time imaging feedback us- 

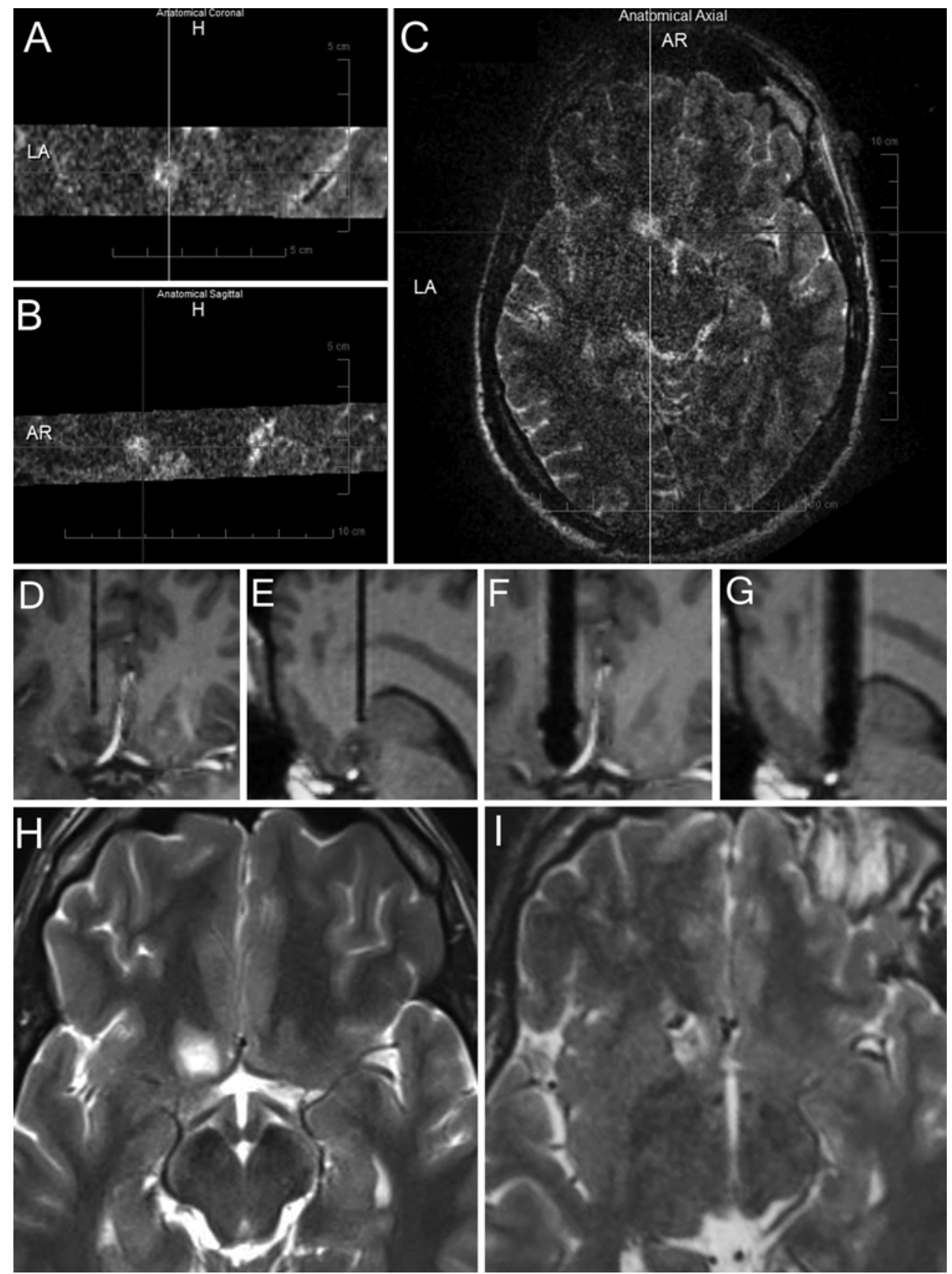

FIG. 4. Case 2. Intraoperative MR images obtained with a 1.5-T scanner. Preoperative coronal (A), sagittal (B), and axial (C) T2-weighted MR images used for trajectory planning. Intraoperative T1-weighted MR images (D-G) demonstrate the surrounding vascular structures and insertion of the ceramic cannula before placement of the biopsy needle. Coronal (D) and sagittal (E) T1-weighted MR images obtained with the tip of the ceramic cannula on the surface of the target lesion. Coronal (F) and sagittal (G) T1-weighted MR images with cutting window of biopsy needle in the target lesion before tissue sampling. Preoperative $(\mathrm{H})$ and postbiopsy (I) T2-weighted MR images demonstrating signal change within the lesion consistent with the removal of tissue at the target.

ing this technique permits on-the-fly targeting adjustments to avoid immediately adjacent vasculature and/or eloquent anatomical structures. Magnetic resonance imaging-confirmed targeting in this manner obviates unnecessary tissue sampling, including frozen section.

\section{Accuracy}

Use of this real-time MRI stereotactic technique and platform allows for reliable and accurate biopsies. Phantom and cadaveric studies using this system for deep brain stimulator lead placement have reported a mean radial error of $0.5 \mathrm{~mm} .{ }^{9}$ Clinical application of similar technology in 29 patients with Parkinson disease who had undergone real-time MRI-guided placement of 53 deep brain stimulation electrodes resulted in a mean radial error of $1.2 \mathrm{~mm}$ and a mean absolute tip error of $2.2 \mathrm{~mm} \cdot{ }^{21}$ Consistent with 
these findings, we found a mean radial error of $1.3 \mathrm{~mm}$ for the final biopsy needle position and a mean absolute tip error of $1.5 \mathrm{~mm}$ (Table 2). These error measurements support the use of our technique for the accurate placement of biopsy needles with a high degree of precision.

\section{Effectiveness}

This real-time MRI-based biopsy technique was effective according to intraoperative MRI confirmation of biopsy needle placement within the lesion, MRI evidence of target sampling, and confirmation of diagnostic tissue on pathological analysis. Because of the accuracy of this biopsy technique, all biopsy samples were taken at the desired target. These resulted in a $100 \%$ diagnostic sampling rate. Furthermore, this skull-mounted MRI guidance technique permitted confirmation and adjustment of the cannula trajectory before placement of the biopsy needle into the brain and/or lesion. While it was not necessary to readjust the trajectory and/or needle depth in any case, on-the-fly adjustments of the cannula trajectory or needle depth could be made, if needed, based on the real-time MRI feedback (Figs. 3 and 4).

\section{Feasibility}

This technique is easily adapted and employed. Average operative time in our cases was 114 minutes. The operative time required for the technique decreased as experience increased. Intraoperative time for individual cases can vary according to the radiographic features of the lesion and the trajectory chosen. Lesion properties can influence the MR sequences selected during planning and the number of targeting adjustments made intraoperatively. The number of scans required to reach the target ranged from 3 to 5 scans per patient in our study. Although pathological diagnosis for unusual lesions may be ambiguous, with MRI confirmation of an accurate biopsy, concern for missing a lesion is eliminated. This renders the frozen section histological analysis unnecessary.

\section{Safety and Clinical Features}

Real-time MRI during stereotactic brain biopsy using this platform is safe and is approved for 1.5-T and compatible with 3.0-T magnets. No patients sustained a clinical or radiographic complication. Cosmetically, the technique described represents a potential improvement over other biopsy platforms in that there is no linear incision requiring sutures or staples. We found that the stab incisions for the mounting screws and twist drill heal quickly with minimal evidence of scarring, less even than that for pins used with the Mayfield head clamp or halo vests. The percutaneous aspect allowed attachment of the frame to the forehead in front of the hairline to optimize the trajectory to the lesion in the patient in Case 2 (Table 1), avoiding neural structures including the ventricle and corpus callosum. Once the targeting is completed, a variety of options beyond biopsy, such as convection-enhanced-delivery infusion cannulae or laser ablation technology, could be applied via the platform with probably similar targeting accuracy and capacity for real-time monitoring of the selected treatment. Although such treatment options are beyond the scope of this paper, they underscore the flexibility of this percutaneous stereotactic iMRI-guided platform.

\section{Conclusions}

Using iMRI real-time biopsy technology offers several advantages. First, it permits standardization of stereotactic MRI-guided biopsies through a percutaneous approach. Second, it allows for safe and precise targeting of brain lesions for biopsy in high-field MR scanners. Third, the defined real-time accuracy assessment of this system can be used in broader scientific applications, including the investigation of intratumoral tissue heterogeneity and correlation with radiographic tumor features.

\section{References}

1. Amin DV, Lozanne K, Parry PV, Engh JA, Seelman K, Mintz A: Image-guided frameless stereotactic needle biopsy in awake patients without the use of rigid head fixation. J Neurosurg 114:1414-1420, 2011

2. Chittiboina P, Heiss JD, Lonser RR: Accuracy of direct magnetic resonance imaging-guided placement of drug infusion cannulae. J Neurosurg 122:1173-1179, 2015

3. Dammers R, Haitsma IK, Schouten JW, Kros JM, Avezaat CJ, Vincent AJ: Safety and efficacy of frameless and framebased intracranial biopsy techniques. Acta Neurochir (Wien) 150:23-29, 2008

4. Dammers R, Schouten JW, Haitsma IK, Vincent AJ, Kros JM, Dirven CM: Towards improving the safety and diagnostic yield of stereotactic biopsy in a single centre. Acta Neurochir (Wien) 152:1915-1921, 2010

5. Dorward NL, Alberti O, Velani B, Gerritsen FA, Harkness WF, Kitchen ND, et al: Postimaging brain distortion: magnitude, correlates, and impact on neuronavigation. J Neurosurg 88:656-662, 1998

6. Hall WA: The safety and efficacy of stereotactic biopsy for intracranial lesions. Cancer 82:1749-1755, 1998

7. Ivan ME, Yarlagadda J, Saxena AP, Martin AJ, Starr PA, Sootsman WK, et al: Brain shift during bur hole-based procedures using interventional MRI. J Neurosurg 121:149160,2014

8. Khatab S, Spliet W, Woerdeman PA: Frameless image-guided stereotactic brain biopsies: emphasis on diagnostic yield. Acta Neurochir (Wien) 156:1441-1450, 2014

9. Larson PS, Starr PA, Bates G, Tansey L, Richardson RM, Martin AJ: An optimized system for interventional magnetic resonance imaging-guided stereotactic surgery: preliminary evaluation of targeting accuracy. Neurosurgery 70 (1 Suppl Operative):95-103, 2012

10. Lobão CA, Nogueira J, Souto AA, Oliveira JA: Cerebral biopsy: comparison between frame-based stereotaxy and neuronavigation in an oncology center. Arq Neuropsiquiatr 67:876-881, 2009

11. Martin AJ, Larson PS, Ostrem JL, Keith Sootsman W, Talke P, Weber OM, et al: Placement of deep brain stimulator electrodes using real-time high-field interventional magnetic resonance imaging. Magn Reson Med 54:1107-1114, 2005

12. Moriarty TM, Quinones-Hinojosa A, Larson PS, Alexander E III, Gleason PL, Schwartz RB, et al: Frameless stereotactic neurosurgery using intraoperative magnetic resonance imaging: stereotactic brain biopsy. Neurosurgery 47:1138-1146, 2000

13. Nauta HJ: Error assessment during "image guided" and "imaging interactive" stereotactic surgery. Comput Med Imaging Graph 18:279-287, 1994

14. Nishihara M, Takeda N, Harada T, Kidoguchi K, Tatsumi $\mathrm{S}$, Tanaka K, et al: Diagnostic yield and morbidity by neuronavigation-guided frameless stereotactic biopsy using magnetic resonance imaging and by frame-based computed tomography-guided stereotactic biopsy. Surg Neurol Int 5 (Suppl 8):S421-S426, 2014 
15. Owen CM, Linskey ME: Frame-based stereotaxy in a frameless era: current capabilities, relative role, and the positiveand negative predictive values of blood through the needle. J Neurooncol 93:139-149, 2009

16. Richardson RM, Kells AP, Martin AJ, Larson PS, Starr PA, Piferi PG, et al: Novel platform for MRI-guided convectionenhanced delivery of therapeutics: preclinical validation in nonhuman primate brain. Stereotact Funct Neurosurg 89:141-151, 2011

17. Richardson RM, Kells AP, Rosenbluth KH, Salegio EA, Fiandaca MS, Larson PS, et al: Interventional MRI-guided putaminal delivery of AAV2-GDNF for a planned clinical trial in Parkinson's disease. Mol Ther 19:1048-1057, 2011

18. Salas S, Brimacombe M, Schulder M: Stereotactic accuracy of a compact intraoperative MRI system. Stereotact Funct Neurosurg 85:69-74, 2007

19. Shieh G: Estimation of the simple correlation coefficient. Behav Res Methods 42:906-917, 2010

20. Sillay KA, Rusy D, Buyan-Dent L, Ninman NL, Vigen KK: Wide-bore 1.5 T MRI-guided deep brain stimulation surgery: initial experience and technique comparison. Clin Neurol Neurosurg 127:79-85, 2014

21. Starr PA, Martin AJ, Ostrem JL, Talke P, Levesque N, Larson PS: Subthalamic nucleus deep brain stimulator placement using high-field interventional magnetic resonance imaging and a skull-mounted aiming device: technique and application accuracy. J Neurosurg 112:479-490, 2010

22. Sumanaweera TS, Adler JR Jr, Napel S, Glover GH: Characterization of spatial distortion in magnetic resonance imaging and its implications for stereotactic surgery. Neurosurgery 35:696-704, 1994

23. Wen DY, Hall WA, Miller DA, Seljeskog EL, Maxwell RE: Targeted brain biopsy: a comparison of freehand computed tomography-guided and stereotactic techniques. Neurosurgery 32:407-413, 1993

24. Willie JT, Laxpati NG, Drane DL, Gowda A, Appin C, Hao $\mathrm{C}$, et al: Real-time magnetic resonance-guided stereotactic laser amygdalohippocampotomy for mesial temporal lobe epilepsy. Neurosurgery 74:569-585, 2014

\section{Disclosure}

The authors report no conflict of interest concerning the materials or methods used in this study or the findings specified in this paper.

\section{Author Contributions}

Conception and design: Elder, Mohyeldin. Acquisition of data: Mohyeldin. Analysis and interpretation of data: all authors. Drafting the article: Mohyeldin. Critically revising the article: all authors. Reviewed submitted version of manuscript: all authors. Statistical analysis: Mohyeldin. Study supervision: Elder.

\section{Correspondence}

J. Bradley Elder, Department of Neurological Surgery, The Ohio State University Wexner Medical Center, 410 W. 10th Ave., Columbus, OH 43210. email: brad.elder@osumc.edu. 